\title{
UNA APROXIMACIÓN AL APRENDIZAJE TOTAL DEL CONCEPTO DE REACCIÓN QUÍMICA
}

Cheyron Castellanos Sánchez, Corporación Universitaria de Ciencias Aplicadas y Ambientales U.D.C.A.

Rómulo Gallego Badillo Universidad Pedagógica Nacional.

\begin{abstract}
In this article, the authors presents a summary of the investigation performed during 1997 and 1998, at the Corporación Universitaria de Ciencias Aplicadas y Ambientales U.D. C.A., based on the design and application of a Pedagogic and Didactic Strategy, for Veterinary Medicine students of first semester, focused in the Teaching and Total Learning of the concept of chemical reaction.
\end{abstract}

\section{RESUMEN}

En este artículo, los autores presentan un resumen de la investigación realizada durante los años de 1997 y 1998, en la Corporación Universitaria de Ciencias Aplicadas y Ambientales U.D.C.A., consistente en el diseño y puesta en marcha de una estrategia pedagógica y didáctica, para estudiantes de primer semestre de Medicina Veterinaria, centrada en la Enseñanza y el Aprendizaje Total del concepto de reacción química.

\section{INTRODUCCION}

Este trabajo de investigación formó parte del Programa de Investigación Representaciones y Conceptos Científicos, dirigido por los profesores Rómulo Gallego Badillo y Royman Pérez Miranda del Departamento de Química, de la Universidad pedagógica Nacional; y surgió del interés que se tiene por la búsqueda de nuevas alternativas en pro del mejoramiento de los procesos de Enseñanza y de Aprendizaje de las Ciencias Experimentales.

El fundamento epistemológico, pedagógico y didáctico de la propuesta, retoma postulados del Constructivismo, Teoría del Caos y Lógica Borrosa; con la intencionalidad de favorecer un aprendizaje total del concepto de reacción química en los estudiantes; es decir, lograr una transformación intelectual de los sujetos desde lo conceptual, lo metodológico, lo actitudinal y lo axiológico (ECMAAs). 
Las investigaciones realizadas en torno a los procesos de Enseñanza y Aprendizaje de las Ciencias Experimentales, han evidenciado que es indispensable introducir modificaciones para posibilitar la construcción de las bases conceptuales propias de cada ciencia; para lo cual es necesario el considerar los diversos factores que inciden de una $u$ otra forma en la construcción de los significados por parte de los estudiantes. Por tal razón, en esta investigación se admitió la aproximación del Aprendizaje Total, cuyo punto de partida es el examen de la propuesta del aprendizaje como cambio conceptual, metodológico, actitudinal y axiológico; donde cada una de estas categorías se codefinen y codeterminan; de tal manera que es imposible interrogarse sobre cada una de ellas sin tener en cuenta las otras (Gallego, etal., 1997).

De otro lado, la revisión de los antecedentes, muestra que la mayoría de investigaciones se han centrado en el proceso Enseñanza-Aprendizaje, desde el punto de vista del cambio conceptual y metodológico. Desde aquí, podría pensarse que el enfoque del aprendizaje total, es una propuesta que proporcionaría resultados más satisfactorios en términos de la eficacia y de la eficiencia de una Enseñanza y de un Aprendizaje de las Ciencias Experimentales.

Se tomó como base el concepto de reacción química ya que muy pocos estudios se han realizado en relación con él, tal vez porque se considera que el aprendizaje de dicho concepto no es nada elemental, sobre todo si no se tienen en cuenta los pensamientos de los alumnos, y porque éstos tienen dificultades incluso en conocimientos que son prerrequisitos para aprenderlo (Carbonell y Furió, 1987). Además los trabajos sobre el concepto de reacción química muestran que muchos estudiantes poseen grandes dificultades para comprender sus aspectos fundamentales, aún al finalizar los estudios de secundaria (Ben-Zvi, 1986; Gabel, 1987 y Meheut, 1989).

\section{INTENCIONALIDADES}

Con este trabajo se pretendió:

- Ordenar el ámbito pedagógico y didáctico, para propiciar la construcción del concepto de reacción química desde la perspectiva del aprendizaje total, en estudiantes de primer semestre de Medicina Veterinaria de la Corporación Universitaria de Ciencias Aplicadas y Ambientales U.D.C.A.

- Identificar las representaciones conceptuales, metodológicas, actitudinales y axiológicas, (ECMAAs), construidas por los estudiantes en relación con el concepto de reacción química.

- Diseñar y validar una estrategia pedagógica y didáctica, que permita transformar las representaciones iniciales que han construido los estudiantes en relación con el concepto de reacción química a unas más próximas a las aceptadas por la comunidad de especialistas.

\section{MARCO CONCEPTUAL}


La epistemología constructivista, en el campo de las Ciencias Experimentales, se ubica dentro de la lógica deductivista (Gallego, 1993). Aquí no se considera que exista el conocimiento previo a la existencia de un sujeto cognoscente; se habla de conocer como una actividad que realizan los sujetos en comunidad, partiendo de sus propias representaciones, dándose un proceso de construcción de significados, de las formas mediante las cuales se elaboran esos significados y de un posicionamiento frente a los mismos.

Desde una postura constructivista, el conocer es una actividad cognoscitiva que realizan lo seres humanos, a partir de las representaciones que ha elaborado sobre sí mismo, sobre la naturaleza y sobre la sociedad; las cuales organiza en estructuras conceptuales, metodológicas, actitudinales y axiológicas (ECMAAs) (Gallego y Pérez, 1994), con la intencionalidad de intervenir y transformar su entorno experiencial. La actividad cognoscitiva como proceso puede comparase a un sistema dinámico no lineal; es decir, que los resultados que ella pueda originar son impredecibles para tiempos largos y cortos; puesto que cada vez que se aprende, las condiciones bajo las cuales se venía comportando el sistema cambian y se hace necesario por ende, variar la trayectoria y las reglas del mismo.

El conocimiento como actividad cognoscitiva, es realizado por los sujetos cognoscentes, en la medida en que se establecen interacciones con los otros, con el medio social, cultural, económico y político; en donde intervienen sus intereses, necesidades e intencionalidades, para forjar su propio proyecto ético de vida. Esta actividad cognoscitiva no puede ser mecánica y repetitiva; sino que debe traducirse en una constante transformación de las ECMAAs.

Dentro de esta perspectiva, se considera una unión entre el constructivismo y la teoría del caos. El constructivismo, desde la concepción de que todo sujeto cognoscente realiza una actividad cognoscitiva a partir de la cual construye su propio saber, que traduce en información para interactuar con los demás miembros de su comunidad. Desde la teoría del caos, en el sentido de que toda actividad cognoscitiva sigue una mecánica no lineal; es decir, que está en constante evolución, por lo que realizar predicciones sobre lo que ella pueda originar resulta prácticamente imposible. Seria ingenuo por tanto creer, que las ECMAAs se podrían contrastar con un orden natural preexistente. Dentro de las Ciencias Experimentales, dicha contrastación se lleva a cabo mediante el experimento.

El marco pedagógico se sustentó en la concepción del aprendizaje total, entendido éste como cambio conceptual, metodológico, actitudinal y axiológico (Gallego y Pérez, 1997). Es importante destacar que si bien el cambio no se da en todos los componentes a la vez, el cambio en uno de ellos determinará las transformaciones que se darán en los demás. El cambio en las ECMAAs, está determinado por la actividad cognoscitiva que cada uno de los sujetos realiza.

Por tanto, en relación con la actividad cognoscitiva, es de esperar que:

- No sea posible predecir los resultados que pueda arrojar (ruptura de causalidad).

- Cada vez que se aprende, las ECMAAs iniciales se transforman (irreversibilidad).

- La actividad cognoscitiva cambia constantemente, por lo que pueden emerger varias soluciones para lo mismo (elección de los puntos de bifurcación).

Del aprendizaje total, pueden destacarse los siguientes aspectos: 
- Cada estudiante atribuye los significados desde sus propias ECMAAs; por lo que la actividad de conocer es un proceso idiosincrático pero que se realza en comunidad.

- La actividad de conocer debe posibilitar el triple reconocimiento en los sujetos; es decir, que cada quien se identifique con lo que ha elaborado, con la forma y el para qué lo ha elaborado.

- El aprendizaje debe tener como finalidad la estructuración del proyecto ético de vida de cada uno de los estudiantes.

- Cada sujeto debe valorar el saber que sabe y el saber de los demás. En este contexto es en donde se realizan las negociaciones mutuas de significados, formas de significar y de actuar.

- A medida que el estudiante va reconstruyendo y construyendo significados, debe hacer partícipes a los demás compañeros y al profesor; transformando en información su saber, con miras a propiciar la controversia necesaria, y establecer acuerdos conceptuales, metodológicos, actitudinales y axiológicos.

- Lo anterior trae como consecuencia la transformación intelectual de los sujetos, la cual se evidencia en el desarrollo de habilidades cognoscitivas y metodológicas, en el cambio de actitud frente a las diversas actividades que se presentan y en la generación de valores como la convivencia, la tolerancia y el respeto entre otros.

El aprendizaje total comprende una interacción del estudiante con lo que él sabe y con las construcciones y reconstrucciones que emprende desde y contra lo que sabe. Al mismo tiempo es una interacción con lo que saben los otros miembros de la comunidad y con el saber que el profesor le presenta. En este proceso se transforman significados, métodos, actitudes y valores; $y$, en la medida en que esto se va dando la forma de aprender cambia, y por tanto las maneras de involucrarse y de comprometer-se. Como consecuencia se deriva que cuando se aprende las estructuras de significado de los sujetos no vuelven a ser las mismas, las formas de aprender cambian, no hay reversibilidad; por eso el aprendizaje total constituye una historia de cambio intelectual global del estudiante, como desenvolvimiento de su proyecto ético de vida.

El concepto de reacción química se caracteriza por ser un concepto científico, de ahí que; el aprendizaje de su significado, se abordó teniendo en cuenta la estructura que un concepto de esta categoría cumple. Dicha estructura se refiere a que un concepto científico debe posibilitar la clasificación, la comparación y la metrización (Mosterín, 1978); además, debe recoger aspectos del objeto de conocimiento propio de cada ciencia, terminar en una formulación matemática, requerir el uso de instrumentos y del diseño de experimentos (Gallego, 1996). Es así como en la Enseñanza y el aprendizaje de este concepto se consideraron aspectos como la conservación de la masa, formación de nuevas sustancias, criterios para predecir la ocurrencia de una reacción química y cinética química entre otros, así como la interpretación cuantitativa de una reacción química y los diversos ensayos experimentales necesarios para abordar la parte conceptual antes mencionada.

De otra parte, lo didáctico se relaciona con el enseñar a leer y a escribir a los estudiantes en el saber que se trate; por lo que la Enseñanza se constituye en un Digitalizado por RED ACADEMICA 
ordenamiento pedagógico y didáctico que hace el profesor con miras a propiciar una experiencia de aprendizaje en los estudiantes. Este ordenamiento consiste en un acto de negociación e intercambio entre el profesor y los estudiantes.

Uno de los propósitos de la Enseñanza universitaria es el de preparar individuos que sean capaces de cumplir sus funciones profesionales; para lo cual, al finalizar sus estudios, deben manejar un vocabulario extenso y flexible, así como poseer un buen nivel de comprensión de los principios básicos de cada ciencia. Lo anterior corresponden a objetivos que no se compaginan bien con una buena parte de las prácticas docentes habituales en las Instituciones de Educación Superior (Muñoz, 1995). La tendencia es la de suministrar gran cantidad de información a los estudiantes exigiendo que sea recordada para un examen, reforzando la noción de que el uso de la memoria es a base del aprendizaje. De ahí que, la Enseñanza universitaria debe promover la actividad del estudiante, su participación e interés; ya que si bien es cierto que el conocimiento teórico con ser importante, no lo es todo en términos de competencia intelectual, y que existen otros procesos intelectuales y habilidades que deben ser desarrollados en los estudiantes.

\section{MARCO METODOLOGICO}

La investigación se desarrolló con 30 estudiantes entre 18 y 22 años de edad, de ambos géneros masculino y femenino, que cursaban primer semestre de Medicina Veterinaria en la U.D.C.A. La problemática motivo de la investigación pretendió establecer en qué medida el ordenamiento del ámbito pedagógico y didáctico orientado hacia la auscultación de la interacción entre los componentes conceptual, metodológico, actitudinal y axiológico; constituyen una aproximación válida para emitir un juicio racional en relación con el aprendizaje total del concepto de reacción química en los estudiantes antes mencionados.

La estrategia pedagógica y didáctica específica consistió en realizar una actividad de motivación, identificar las representaciones iniciales de los estudiantes en relación con el concepto de reacción química, y propiciar la transformación de dichas representaciones cuyo significado sea lo más próximo a lo aceptado por la comunidad de especialistas.

Los instrumentos empleados fueron:

- Cuestionario de 4 preguntas, para identificar las representaciones de los estudiantes en relación con los conceptos de reacción química, ecuación química, reactivo y producto.

- Diseño de 13 Actividades Experimentales, para identificar e iniciar la transformación en las ECMAAs de cada uno de los estudiantes. En estas actividades el concepto de reacción química se abordó desde el punto de vista de conservación de la masa, los diversos efectos producidos durante el transcurso de la misma, formación de nuevas sustancias y cinética química.

- Mapas conceptuales, que fueron empleados durante todo el proceso como mecanismo para establecer el progreso en la construcción de los significados, formas de significar y de actuar de los estudiantes.

- Prueba tipo likert para auscultar la Interacción entre los componentes conceptual, metodológico, actitudinal y axiológico. 
- Juicios de valor, como herramienta para posibilitar el triple reconocimiento en cada uno de los estudiantes.

\section{RESULTADOS Y DISCUSIÓN}

Para realizar la discusión de resultados, cada una de las representaciones elaboradas por los estudiantes se clasificó teniendo en cuenta la existencia o no de un modelo explicativo coherente, el uso de lenguaje científico o de tipo cotidiano y la aproximación de los significados elaborados por ellos a los aceptados por la comunidad de especialistas.

Cuestionario. Para la gran mayoría de estudiantes el significado del concepto de reacción química es sinónimo de mezcla, el de ecuación química lo relacionan con la resolución de problemas químicos, el de reactivo como la sustancia necesaria para realizar un experimento y el de producto como el resultado de una ecuación o experimento; por lo que puede considerarse que dichas representaciones constituyen concepciones alternativas en las que no se evidencia una diferenciación progresiva y una reconciliación integradora entre estos conceptos.

El lenguaje utilizado para expresar las ideas es de tipo cotidiano, muy pocos estudiantes hacen uso de lenguaje científico, y en general se evidencia la falta de un modelo explicativo coherente, que permita acercarse a lo aceptado por la comunidad de especialistas.

Se asume que las representaciones construidas son "ingenuas", entendiéndose por ello, que carecen de rigor científico.

Actividades experimentales. Durante el desarrollo de las diversas actividades experimentales, se pudo establecer como los estudiantes dan respuestas apresuradas basadas en los cambios que son visibles a la percepción. Inicialmente, la mayoría de estudiantes clasificaban como procesos químicos aquellos en los que podían observar cambios en la coloración o el desprendimiento de un gas. Sin embargo, durante y al finalizar el proceso, con estas actividades se pudo lograr una transformación en las ECMAAs de un número significativo de estudiantes, ya que consideraban indispensable el uso de instrumentos para determinar la formación de nuevas sustancias y que no todos los cambios que ocurren durante el transcurso de la misma son observables.

Mapas conceptuales. El análisis de los mapas conceptuales se realizó desde una perspectiva cualitativa. Al respecto es importante anotar que la construcción de los mapas conceptuales representó serias dificultades para todos los estudiantes, ya que no estaban familiarizados con este tipo de trabajo. En los mapas iniciales que construyeron los estudiantes, se evidenciaron algunas proposiciones incoherentes, la permanencia de concepciones alternativas basadas en el uso de lenguaje cotidiano y la carencia de relaciones cruzadas. Durante el transcurso del proceso, algunos estudiantes mostraron avances significativos tanto en la construcción de los mapas conceptuales, como en la transformación de significados y formas de significar en relación con el concepto de reacción química.

Al final del proceso, en los mapas construidos por cada estudiante fue palpable la presencia de un modelo explicativo, que les permitió explicar e interpretar cada una de las Digitalizado por RED ACADEMICA 
temáticas. Sin embargo, muy pocos estudiantes no alcanzaron con satisfacción los logros, en lo que a ésta actividad de aprendizaje se refiere.

Prueba tipo Likert y Juicios de valor. En cuanto a la prueba tipo likert, al inicio del proceso los estudiantes muestran una posición de rechazo frente a lo conceptual y lo metodológico, mientras que en lo actitudinal y axiológico la tendencia es más neutral, evidenciándose una aparente ruptura entre estos componentes. Al finalizar el proceso y volver a aplicar la prueba, la transformación en las ECMAAs así como la interacción entre los componentes, es evidente para la mayoría de estudiantes. Se evidenció por tanto, que en la medida en que se transformaba cualquiera de los aspectos que conforman la estructura de significado de un sujeto, se inicia el proceso de transformación de los otros componentes, por lo que resulta válido afirmar la permanente y mutua interacción e interrelación entre lo conceptual, lo metodológico, lo actitudinal y lo axiológico.

Por otra parte, los juicios de valor se consolidaron como instrumentos válidos, en la medida en que posibilitaron que cada estudiante fuera consciente de su progreso, identificara sus fortalezas y así mismo sus debilidades. Posibilitaron además, que cada uno se comprometiera con su propio aprendizaje. El generar espacios de autorreflexión, permiten determinar las fallas y los aciertos, de manera que si es necesario introducir transformaciones se puedan realizar. De igual manera, es una herramienta eficaz para que cada estudiante sea consciente de su propio trabajo y esfuerzo, reconozca que es él el artífice de su aprendizaje, y de que tome conciencia frente a su transformación intelectual.

\section{CONCLUSIONES}

- Las representaciones iniciales construidas por los estudiantes en relación con el concepto de reacción química, consistían en concepciones alternativas cuyo significado se encontraba muy alejado del aceptado por la comunidad de especialistas.

- Al igual que las consideraciones de Carbonell y Furió (1987), y las de Landau y Lastres (1996), se comparte la premisa de que para los estudiantes resulta un tanto complejo comprender el significado del concepto de reacción química.

- La estrategia diseñada fue eficaz, ya que para la mayoría de estudiantes se posibilitó una transformación de las ECMAAs logrando un cambio en el significado del concepto de reacción química. De ahí que, puede afirmarse que el ordenamiento del ámbito pedagógico y didáctico propició el aprendizaje total del concepto de reacción química en los estudiantes. No obstante, es importante señalar que a pesar de la instrucción y de la realización de las diversas actividades tanto experimentales como de aula, al final del proceso, persiste en pocos estudiantes la concepción de que una reacción química es la mezcla de dos sustancias.

- Los instrumentos utilizados permitieron por una parte, identificar las representaciones de los estudiantes, y por otra, posibilitar la transformación de las mismas.

- En términos generales, se establece que de acuerdo con los resultados obtenidos, la teoría del aprendizaje total es sostenible, en la medida en que se propició la transformación intelectual de los sujetos involucrados en el proceso, desde lo conceptual, 
lo metodológico, lo actitudinal y lo axiológico. De igual manera, se determinó que los intereses y el proyecto de vida de cada quien, orientan el proceso de construcción de significados, formas de significar y de actuar. Por otra parte, la complejidad inherente a la actividad cognoscitiva, no posibilita establecer generalizaciones temporales ni espaciales; por lo que los resultados de dicha actividad se evidenciarán en la medida en que se ponga en consideración de los demás las elaboraciones que cada uno de los sujetos vaya construyendo, y se realicen los procesos de negociación y establecimiento de acuerdos.

\section{BIBLIOGRAFÍA}

BEN-ZVI, R., et al. 1986. Is in atom of copper malleable? In: Journal of Chemical Education. No. 63. Pp. 64-66.

CARBONELL, F. y FURIÓ. C. 1987. "Opiniones de los adolescentes respecto al cambio sustancia en las reacciones químicas". En: Enseñanza de las Ciencias. Vol. 5. No. 1. Pp. 3-9.

GABEL, D. 1987. Let us go back to nature study. In: Journal of Chemical Education. Vol. 64. No. 9. Pp. 727-729.

GALLEGO, R. 1993. Discurso sobre el constructivismo. Santafé de Bogotá. Cooperativa Editorial Magisterio.

GALLEGO, R. 1996. Discurso constructivista sobre las ciencias experimentales. Una concepción actual sobre el conocimiento científico. Santafé de Bogotá. Cooperativa Editorial Magisterio.

GALLEGO, R. y PÉREZ, R. 1994. Representaciones y conceptos científicos. Un programa de investigación. Santafé de Bogotá. Universidad Pedagógica Naciona. Departamento de Química.

GALLEGO, R. y PÉREZ, R. 1997. El concepto de Aprendizaje Total: Una aproximación. Santafé de Bogotá. Universidad Pedagógica Nacional. Departamento de Química.

LANDAU, L. y LASTRES. L. 1996. Cambios químicos y conservación de la masa... ¿Está todo claro? En: Enseñanza de las Ciencias. Vol 14. No 2. Pág. 171-174.

MOSTERÍN, J. 1978. La estructura de los conceptos científicos. En: Investigación y ciencia. No. 16. Pp. 82-93.

MUÑOZ, CH: : "Escribir para aprender: Ensayo de una alternativa en la Enseñanza universitaria de las ciencias”. En. Enseñanza de las Ciencias. Vol. 12. No. 1. Págs. 97100. 